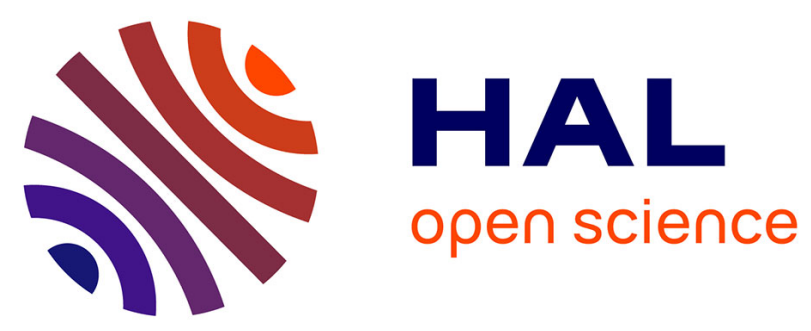

\title{
A multislope MUSCL method on unstructured meshes applied to compressible Euler equations for axisymmetric swirling flows
}

Stéphane Clain, David Rochette, Rachid Touzani

\section{- To cite this version:}

Stéphane Clain, David Rochette, Rachid Touzani. A multislope MUSCL method on unstructured meshes applied to compressible Euler equations for axisymmetric swirling flows. Journal of Computational Physics, 2010, 229 (13), pp.4884-4906. 10.1016/j.jcp.2010.03.004 hal-00340087

\section{HAL Id: hal-00340087 \\ https://hal.science/hal-00340087}

Submitted on 19 Nov 2008

HAL is a multi-disciplinary open access archive for the deposit and dissemination of scientific research documents, whether they are published or not. The documents may come from teaching and research institutions in France or abroad, or from public or private research centers.
L'archive ouverte pluridisciplinaire HAL, est destinée au dépôt et à la diffusion de documents scientifiques de niveau recherche, publiés ou non, émanant des établissements d'enseignement et de recherche français ou étrangers, des laboratoires publics ou privés. 


\title{
A multislope MUSCL method on unstructured meshes applied to compressible Euler equations for swirling flows
}

\author{
S. Clain ${ }^{\text {a }}$, D. Rochette ${ }^{\mathrm{b}}$, R. Touzani ${ }^{\mathrm{c}}$ \\ a Institut de Mathématiques, CNRS UMR 5219, Université Paul Sabatier Toulouse \\ 3, 118 route de Narbonne, 31062 Toulouse Cedex 4, France. \\ ${ }^{\mathrm{b}}$ Laboratoire Arc Électrique et Plasmas Thermiques, CNRS FRE 3120, Université \\ Blaise Pascal - IUT de Montluçon, Avenue Aristide Briand, BP 2235, 03101 \\ Montluçon Cedex, France. \\ ${ }^{\mathrm{c}}$ Laboratoire de Mathématiques, CNRS UMR 6620, Université Blaise Pascal \\ (Clermont-Ferrand II), Campus universitaire des Cézeaux,t 63177 Aubière Cedex, \\ France.
}

\begin{abstract}
A finite volume method for the numerical solution of axisymmetric inviscid swirling flows is presented. The governing equations of the flow are the axisymmetric compressible Euler equations including swirl (or tangential) velocity. A first-order scheme is introduced. In this one, convective fluxes at cell interfaces are evaluated by the Rusanov or the HLLC numerical flux and geometric source terms are discretized by the explicit Euler method. Extension to the second-order space approximation using a multislope MUSCL method is derived. A stationary solution of the fluid flow following the radial direction has been established with a zero and non-zero tangential velocity. Numerical and exact solutions are compared for the Riemann problem. Effectiveness of the multislope MUSCL scheme is demonstrated for strongly shocked axially symmetric flows as the forward-facing step and the spherical bubble compression problems.
\end{abstract}

Key words: Axisymmetric compressible Euler equations, Swirling flow, Finite volumes, MUSCL method, Unstructured mesh.

\section{Introduction}

Axisymmetric Euler system using cylindrical coordinates is used in numerous applications such as axisymmetric flows in a nozzle [1], supersonic jets [2], turbomachine modelling [3,4]. More recently, inductive plasma flows are modelled 
with the axisymmetric Euler formulation taking into account the tangential velocity, the so-called swirling flow $[5,6]$.

From a numerical point of view, the finite volume method [7-9] is a popular technique to compute numerical approximations of the Euler system solution for axisymmetric geometries. A particular issue concerns the choice of the variables to conserve. In a first approach, the mean value approximation of any generic function $v$ on the cell $C_{i}$ is performed by using the classical average

$$
v_{i} \approx \frac{\int_{C_{i}} v d r d z}{\int_{C_{i}} d r d z}
$$

where we employ the measure (metric) $d r d z$ [10]. A second approach consists in computing the mean value using the measure $r d r d z$ [7]:

$$
v_{i} \approx \frac{\int_{C_{i}} v r d r d z}{\int_{C_{i}} r d r d z}
$$

which leads to a better formulation. Indeed, $v_{i}$ corresponds to the mean value of $v$ in the three-dimensional context, i.e. the mean value on the axisymmetric torus with section $C_{i}$. On the other hand, the formulation simplifies the boundary condition at $r=0$ since a null flux value naturally derives from the flux integration and no additional constraint is required on the symmetry axis [7].

To provide an approximation of the solution of the axisymmetric Euler system, we use a fractional step technique where we split the formulation into a conservative homogeneous equation and the source term. To solve the conservative part, the traditional technique consists in using a first-order solver (Roe, HLLC or Riemann solver) combined with a second-order reconstruction such as the MUSCL method [11,12] to improve accuracy. The classical MUSCL technique uses a piecewise linear reconstruction with a slope limiting procedure to ensure the $L^{\infty}$-stability. Then two new approximations are computed on both sides of each edge and are employed in the numerical flux evaluation. We propose here to use a new reconstruction technique: the multislope MUSCL method [13-15] where the reconstructed values are obtained using an approximation of specific directional derivatives instead of the full gradient. The main advantage is that the reconstruction can be rewritten as a one-dimensional MUSCL method at each interface leading to a simple and efficient scheme.

In the axisymmetric context, there are few numerical tests to validate the scheme for compressible Euler equations. For example, we are not able to compute the exact solution of the Riemann problem excepting in very particular situations. We propose a new numerical test for the swirling flow based 
on the steady-state situation. We manage to reduce the Euler system to an ordinary differential equation and a simple approximation based the Euler forward algorithm is proposed to provide an accurate numerical solution.

The organization of the paper is as follows. In section 2, we present the governing Euler equations in cylindrical coordinates assuming rotational symmetry. In section 3, we present the numerical scheme and its second-order extension using a multislope MUSCL method. In section 4, we establish a stationary solution assuming that the flow depends only on the radial direction. Finally, we present numerical experiments to test the obtained scheme.

\section{Axisymmetric Euler equations for swirling flows}

We first present compressible Euler equations using the cylindrical coordinates and simplify them under the axisymmetric invariance assumption. For any point $X=(x, y, z) \in \mathbb{R}^{3}$ we denote by $(r, \theta, z)$ the associated cylindrical coordinates. Let $\mathcal{P}=\mathbb{R}^{+} \times \mathbb{R}=\{r \geq 0, z \in \mathbb{R}\}$ denote an axial cut of the three dimensional space (the set of parameters) and let $\Omega$ be an open set of $\mathcal{P}$. The open set $\widetilde{\Omega} \subset \mathbb{R}^{3}$ will denote the three-dimensional volume obtained by rotation around the axial direction $0 z$, i.e.

$$
\widetilde{\Omega}:=\{(r \cos \theta, r \sin \theta, z) ;(r, z) \in \Omega, 0 \leq \theta<2 \pi\} .
$$

We start by giving the compressible Euler equations in the domain $\widetilde{\Omega}$ using cartesian coordinates:

$$
\begin{aligned}
& \frac{\partial \rho}{\partial t}+\nabla \cdot(\rho \mathbf{u})=0 \\
& \frac{\partial(\rho \mathbf{u})}{\partial t}+\nabla \cdot(\rho \mathbf{u} \otimes \mathbf{u}+P \mathbf{I})=0 \\
& \frac{\partial E}{\partial t}+\nabla \cdot((E+P) \mathbf{u})=0
\end{aligned}
$$

where $\rho$ is the fluid mass density, $P$ is the pressure, $\mathbf{u}$ is the velocity vector and $E$ is the total energy per unit volume. The tensors $\mathbf{u} \otimes \mathbf{u}$ and $\mathbf{I}$ stand for the tensor product of $\mathbf{u}$ by $\mathbf{u}$ and the unit tensor respectively.

To close the system, we add a state equation which in general form reads

$$
P=\hat{P}(\rho, e)
$$


where $e$ stands for the specific internal energy related to the total energy by:

$$
E=\rho e+\frac{1}{2} \rho|\mathbf{u}|^{2} .
$$

In the sequel, we restrict ourselves to an ideal gas, that is,

$$
P=(\gamma-1) \rho e,
$$

where $\gamma$ is the ratio of specific heats at constant pressure and volume.

We introduce the mapping defining cylindrical coordinates:

$$
(r, \theta, z) \mapsto(r \cos \theta, r \sin \theta, z)
$$

To any scalar function $f: \widetilde{\Omega} \rightarrow \mathbb{R}$, we associate the function $\widetilde{f}(r, \theta, z)=$ $f(r \cos \theta, r \sin \theta, z)$ for $(r, z) \in \Omega$ and $\theta \in[0,2 \pi)$. Due to the axial symmetry we look for solutions (density, velocity, pressure, ...) that are independent of $\theta$, i.e. all the involved functions will depend on space variables $r$ and $z$ and on the time variable $t$.

In the sequel, for the sake of simplicity, we shall omit the tilde symbol on the functions $f=f(r, z)$. Applying the divergence operator in cylindrical coordinates and eliminating the $\theta$-derivatives, we deduce from (1)-(3) the following system:

$$
\begin{aligned}
& \frac{\partial \rho}{\partial t}+\frac{1}{r} \frac{\partial}{\partial r}\left(r \rho u_{r}\right)+\frac{\partial}{\partial z}\left(\rho u_{z}\right)=0, \\
& \frac{\partial}{\partial t}\left(\rho u_{r}\right)+\frac{\partial}{\partial r}\left(\rho u_{r}^{2}+P\right)+\frac{\partial}{\partial z}\left(\rho u_{r} u_{z}\right)+\frac{1}{r} \rho\left(u_{r}^{2}-u_{\theta}^{2}\right)=0, \\
& \frac{\partial}{\partial t}\left(\rho u_{z}\right)+\frac{\partial}{\partial r}\left(\rho u_{z} u_{r}\right)+\frac{\partial}{\partial z}\left(\rho u_{z}^{2}+P\right)+\frac{1}{r} \rho u_{z} u_{r}=0, \\
& \frac{\partial}{\partial t}\left(\rho u_{\theta}\right)+\frac{\partial}{\partial r}\left(\rho u_{\theta} u_{r}\right)+\frac{\partial}{\partial z}\left(\rho u_{\theta} u_{z}\right)+\frac{2}{r} \rho u_{\theta} u_{r}=0, \\
& \frac{\partial E}{\partial t}+\frac{1}{r} \frac{\partial}{\partial r}\left(r u_{r}(E+P)\right)+\frac{\partial}{\partial z}\left(u_{z}(E+P)\right)=0 .
\end{aligned}
$$

Note that assuming rotational symmetry does not reduce the number of unknowns since the velocity field has three nonzero components that are respectively the radial $u_{r}(r, z)$, the tangential $u_{\theta}(r, z)$ and the axial component $u_{z}(r, z)$.

To derive a conservative form of the system, we multiply equations (5)-(9) by the radial coordinate $r$ like in $[16,8]$. We then obtain the final form of the Euler equations: 


$$
\begin{aligned}
& \frac{\partial}{\partial t}(r \rho)+\frac{\partial}{\partial r}\left(r \rho u_{r}\right)+\frac{\partial}{\partial z}\left(r \rho u_{z}\right)=0 \\
& \frac{\partial}{\partial t}\left(r \rho u_{r}\right)+\frac{\partial}{\partial r}\left(r \rho u_{r}^{2}+r P\right)+\frac{\partial}{\partial z}\left(r \rho u_{r} u_{z}\right)=\rho u_{\theta}^{2}+P, \\
& \frac{\partial}{\partial t}\left(r \rho u_{z}\right)+\frac{\partial}{\partial r}\left(r \rho u_{z} u_{r}\right)+\frac{\partial}{\partial z}\left(r \rho u_{z}^{2}+r P\right)=0, \\
& \frac{\partial}{\partial t}\left(r \rho u_{\theta}\right)+\frac{\partial}{\partial r}\left(r \rho u_{\theta} u_{r}\right)+\frac{\partial}{\partial z}\left(r \rho u_{\theta} u_{z}\right)=-\rho u_{\theta} u_{r}, \\
& \frac{\partial}{\partial t}(r E)+\frac{\partial}{\partial r}\left(r u_{r}(E+P)\right)+\frac{\partial}{\partial z}\left(r u_{z}(E+P)\right)=0 .
\end{aligned}
$$

Equivalently, we can write this system under the conservative form:

$$
\frac{\partial(r U)}{\partial t}+\frac{\partial\left(r F_{r}(U)\right)}{\partial r}+\frac{\partial\left(r F_{z}(U)\right)}{\partial z}=G(U)
$$

where $U$ is the conservative variable vector defined by

$$
U=\left(\begin{array}{c}
\rho \\
\rho u_{r} \\
\rho u_{z} \\
\rho u_{\theta} \\
E
\end{array}\right)
$$

and the fluxes $F_{r}(U)$ and $F_{z}(U)$ and the geometric source term $G(U)$ are given by:

$$
F_{r}(U)=\left(\begin{array}{c}
\rho u_{r} \\
\rho u_{r}^{2}+P \\
\rho u_{z} u_{r} \\
\rho u_{\theta} u_{r} \\
u_{r}(E+P)
\end{array}\right), \quad F_{z}(U)=\left(\begin{array}{c}
\rho u_{z} \\
\rho u_{r} u_{z} \\
\rho u_{z}^{2}+P \\
\rho u_{\theta} u_{z} \\
u_{z}(E+P)
\end{array}\right), \quad G(U)=\left(\begin{array}{c}
0 \\
\rho u_{\theta}^{2}+P \\
0 \\
-\rho u_{\theta} u_{r} \\
0
\end{array}\right) .
$$

Clearly, the formulation (15) involves a divergence form in the left-hand side and the remaining terms are considered as source terms on the right-hand side. This form of the equations enables casting the so-called well-balanced numerical schemes required to enable preserving equilibrium states like gas at rest. 


\section{A finite volume scheme with multislope MUSCL reconstruction}

\subsection{A first-order finite volume scheme}

To address numerical approximation, we describe the finite volume method we use for the conservative part of the Euler system. We consider a conforming unstructured mesh $\mathcal{T}_{h}$ of the two-dimensional domain $\Omega$, made of triangles (or cells) $C_{i} \subset \Omega, i=1, \ldots, I$. We denote by $\nu(i)$ the index set of the neighbouring triangles $C_{j}$ which share a common edge $S_{i j}$ with the cell $C_{i}$ and by $\mathbf{n}_{i j}=$ $\left(n_{i j, r}, n_{i j, z}\right)$ the outward unit normal vector to $C_{i}$.

We first integrate the system (15) over the cell $C_{i}$ and use the Green formula to get

$$
\frac{d}{d t} \int_{C_{i}} U(r, z, t) r d r d z+\int_{\partial C_{i}}\left(F_{r}(U) n_{i j, r}+F_{z}(U) n_{i j, z}\right) r d \sigma=\int_{C_{i}} G(U) d r d z
$$

where $\partial C_{i}$ denotes the boundary of the cell $C_{i}$. Let $\left(t^{n}=n \Delta t\right)_{n \in \mathbb{N}}$ be a uniform subdivision of the time interval $[0,+\infty)$. Integrating on the interval $\left[t^{n}, t^{n+1}\right]$ we obtain the equation:

$$
\begin{aligned}
\int_{C_{i}} U\left(r, z, t^{n+1}\right) r d r d z= & \int_{C_{i}} U\left(r, z, t^{n}\right) r d r d z \\
& -\int_{t^{n}}^{t^{n+1}} \int_{\partial C_{i}}\left(F_{r}(U) n_{i j, r}+F_{z}(U) n_{i j, z}\right) r d \sigma d t \\
& +\int_{t^{n}}^{t^{n+1}} \int_{C_{i}} G(U) d r d z d t .
\end{aligned}
$$

We aim at computing a constant piecewise approximation of the solution $U$ on each cell $C_{i}$. To this end, $U_{i}^{n}$ represents an approximation of the average of $U$ on $C_{i}$ at time $t^{n}$. More precisely, for a given cell $C_{i}$, we introduce the following measures

$$
\left|C_{i}\right|=\int_{C_{i}} d r d z, \quad\left|C_{i}\right|_{r}=\int_{C_{i}} r d r d z
$$

and we define the approximation

$$
U_{i}^{n} \approx \frac{1}{\left|C_{i}\right|_{r}} \int_{C_{i}} U\left(r, z, t^{n}\right) r d r d z
$$


In the same way, we define the measures of an edge

$$
\left|S_{i j}\right|=\int_{S_{i j}} d \sigma, \quad\left|S_{i j}\right|_{r}=\int_{S_{i j}} r d \sigma
$$

and we define the approximation of the flux across the interface $S_{i j}$ during the interval $\left[t^{n}, t^{n+1}\right]$ by

$$
F_{i j}^{n} \approx \frac{1}{\Delta t\left|S_{i j}\right|_{r}} \int_{t^{n}}^{t^{n+1}} \int_{S_{i j}}\left(F_{r}(U) n_{i j, r}+F_{z}(U) n_{i j, z}\right) r d \sigma d t .
$$

We eventually define an approximation of the right-hand side contribution by

$$
G_{i}^{n} \approx \frac{1}{\Delta t\left|C_{i}\right|} \int_{t^{n}}^{t^{n+1}} \int_{C_{i}} G(U) d r d z d t
$$

It results from (15) that the integrals involve two kinds of measures: $r d r d z$ and $d r d z$. To compute the mean value on the cell $C_{i}$ employing the $r d r d z$ measure, we have to divide by $\left|C_{i}\right|_{r}$ since the approximation (16) becomes an equality for constant functions. On the other hand, we divide by $\left|C_{i}\right|$ to provide a mean value of $G_{i}^{n}$ since we use the $d r d z$ measure in the integral (see [7], p. 495).

To define an explicit scheme, we use a numerical flux such that

$$
F_{i j}^{n}=\mathcal{F}\left(U_{i}^{n}, U_{j}^{n}, \mathbf{n}_{i j}\right), \quad G_{i}^{n}=G\left(U_{i}^{n}\right) .
$$

The scheme reads then

$$
\left|C_{i}\right|_{r} U_{i}^{n+1}=\left|C_{i}\right|_{r} U_{i}^{n}-\Delta t \sum_{j \in \nu(i)}\left|S_{i j}\right|_{r} \mathcal{F}\left(U_{i}^{n}, U_{j}^{n}, \mathbf{n}_{i j}\right)+\Delta t\left|C_{i}\right| G\left(U_{i}^{n}\right) .
$$

In the present study, we present two numerical fluxes. We first use the Rusanov flux

$$
\mathcal{F}\left(U_{i}, U_{j}, \mathbf{n}_{i j}\right)=\frac{F_{r}\left(U_{i}\right)+F_{r}\left(U_{j}\right)}{2} n_{i j, r}+\frac{F_{z}\left(U_{i}\right)+F_{z}\left(U_{j}\right)}{2} n_{i j, z}-\lambda_{i j}\left(U_{j}-U_{i}\right)
$$

with $\lambda_{i j}=\lambda\left(U_{i}, U_{j}\right)$ large enough to ensure stability (see [16]). The main advantage of such a flux is its ability to handle real gases. The drawbacks are on one hand a significant diffusion amount and, on the other hand, contact discontinuities are not well approximated when steady-state problems are considered (see [16] p. 293, [17], and [18] p. 204). We then resort to a second less 
diffusive numerical flux: the HLLC flux (see [16] for a technical description). For this one, we obtain a better resolution of the contact discontinuity for stationary solutions while flux computations remain reasonable for complex applications (real gas, multispecies and multiphase problems for instance).

Let us give a new expression of (17) that uses the $\left|C_{i}\right|$ and $\left|S_{i j}\right|$ measures only. For a given triangle $C_{i}$, let $\left(r_{i, 1}, z_{i, 1}\right),\left(r_{i, 2}, z_{i, 2}\right),\left(r_{i, 3}, z_{i, 3}\right)$ stand for the coordinates of its three vertices. A similar notation is used for a given edge $S_{i j}$. Using the identities

$$
\left|C_{i}\right|_{r}=\left|C_{i}\right| \frac{\left(r_{i, 1}+r_{i, 2}+r_{i, 3}\right)}{3}, \quad\left|S_{i j}\right|_{r}=\left|S_{i j}\right| \frac{\left(r_{i j, 1}+r_{i j, 2}\right)}{2},
$$

we obtain from (17),

$$
\begin{aligned}
U_{i}^{n+1}=U_{i}^{n} & -\Delta t \sum_{j \in \nu(i)} \frac{\left|S_{i j}\right|}{\left|C_{i}\right|} \frac{3\left(r_{i j, 1}+r_{i j, 2}\right)}{2\left(r_{i, 1}+r_{i, 2}+r_{i, 3}\right)} \mathcal{F}\left(U_{i}^{n}, U_{j}^{n}, \mathbf{n}_{i j}\right) \\
& +\frac{3 \Delta t}{r_{i, 1}+r_{i, 2}+r_{i, 3}} G\left(U_{i}^{n}\right) .
\end{aligned}
$$

This new formulation enables proving that the numerical scheme preserves the situation when the gas is at rest. Indeed, consider the initial condition $U^{0}$ where the pressure $P^{0}$ and the density $\rho^{0}$ are constant and the velocity is null, the resulting numerical flux is given by

$$
\mathcal{F}\left(U_{i}^{0}, U_{j}^{0}, \mathbf{n}_{i j}\right)=\left(\begin{array}{c}
0 \\
P^{0} n_{i j, r} \\
P^{0} n_{i j, z} \\
0 \\
0
\end{array}\right) .
$$

We easily deduce that we have after the first step $\rho_{i}^{1}=\rho^{0}, u_{\theta, i}^{0}=u_{\theta, i}^{1}=0$ and $E_{i}^{1}=E^{0}$ since the first and the two last components of vector $G$ are null.

It remains to study radial and axial components of the velocity $u_{r, i}^{1}$ and $u_{z, i}^{1}$. From the momentum equations, the flux contribution is

$$
\mathbf{C}_{\text {flux }}=\sum_{j \in \nu(i)} \frac{\left|S_{i j}\right|}{\left|C_{i}\right|} \frac{3\left(r_{i j, 1}+r_{i j, 2}\right)}{2\left(r_{i, 1}+r_{i, 2}+r_{i, 3}\right)} P^{0} \mathbf{n}_{i j}
$$




$$
\begin{aligned}
& =\frac{P^{0}}{\left|C_{i}\right|_{r}} \sum_{j \in \nu(i)}\left|S_{i j}\right|_{r} \mathbf{n}_{i j} \\
& =\frac{P^{0}}{\left|C_{i}\right|_{r}} \sum_{j \in \nu(i)} \int_{S_{i j}} r \mathbf{n}_{i j} d \sigma .
\end{aligned}
$$

Using the Green formula we obtain

$$
\mathbf{C}_{\text {flux }}=\frac{P^{0}}{\left|C_{i}\right|_{r}} \int_{C_{i}} \nabla_{r, z}(r) d r d z=P^{0} \frac{\left|C_{i}\right|}{\left|C_{i}\right|_{r}}\left(\begin{array}{l}
1 \\
0
\end{array}\right) .
$$

The right-hand side term for the radial and axial equations reduces to

$$
\mathbf{C}_{\mathrm{rhs}}=\frac{3}{r_{i, 1}+r_{i, 2}+r_{i, 3}} P^{0}\left(\begin{array}{l}
1 \\
0
\end{array}\right)=\frac{\left|C_{i}\right|}{\left|C_{i}\right|_{r}} P^{0}\left(\begin{array}{l}
1 \\
0
\end{array}\right) .
$$

Since $\mathbf{C}_{\text {flux }}=\mathbf{C}_{\mathrm{rhs}}$, then $u_{r, i}^{n}=u_{z, i}^{n}=0$ and the gas stays at rest.

\subsection{A second-order scheme using the multislope MUSCL method}

In the early 70's, Van Leer [11] introduced the MUSCL technique (Monotonic Upwind Schemes for Conservation Laws) to get a more accurate approximation with less diffusion effect while maintaining stability. Extensions to multidimensional situations for unstructured meshes have been proposed (see [12,18]). We present here a new extension of the MUSCL technique on triangles where we use approximations of the directional derivative of $U$ as proposed in [13-15] instead of an approximation of $\nabla U$.

The goal is to compute a more accurate flux $\mathcal{F}\left(U_{i j}^{n}, U_{j i}^{n}, \mathbf{n}_{i j}\right)$ where $U_{i j}^{n}$ and $U_{j i}^{n}$ are better approximations of $U$ on both sides of the edge $S_{i j}$. In the following, we detail the method to construct the $U_{i j}$ values where we skip the time index $n$ for the sake of simplicity.

\subsubsection{The fundamental decomposition}

For a given volume $C_{i}$, we denote by $\mathbf{B}_{i}$ the centroid and $\mathbf{Q}_{i j}$ the intersection of the segment $\left[\mathbf{B}_{i}, \mathbf{B}_{j}\right]$ with the common edge $S_{i j}$ for all $j \in \nu(i)$ (see figure. $1)$. 


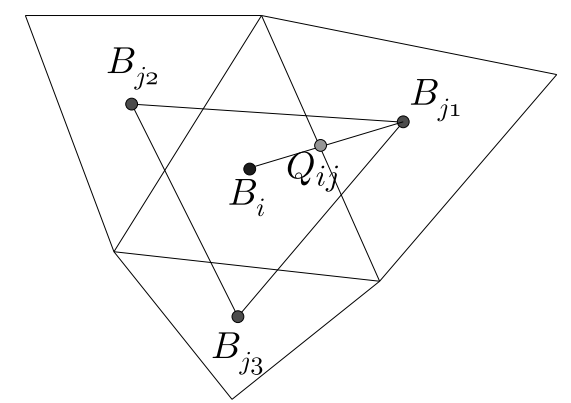

Fig. 1. Geometrical ingredients and notations.

We introduce the barycentric coordinates $\left(\rho_{i j}\right)_{j \in \nu(i)}$, defined by

$$
\sum_{j \in \nu(i)} \rho_{i j} \mathbf{B}_{j}=\mathbf{B}_{i}, \quad \sum_{j \in \nu(i)} \rho_{i j}=1 .
$$

We assume that the point $\mathbf{B}_{i}$ is strictly inside the triangle formed by the three other points. Therefore $\rho_{i j}>0$. Defining the normalized direction

$$
\mathbf{t}_{i j}=\frac{\mathbf{B}_{i} \mathbf{B}_{j}}{\left|\mathbf{B}_{i} \mathbf{B}_{j}\right|}
$$

and using relation (18), we obtain a decomposition (referred to as the fundamental decomposition) of $t_{i j}$ in function of the two other directions:

$$
\mathbf{t}_{i j}=\sum_{\substack{k \in \nu(i) \\ k \neq j}} \beta_{i j k} \mathbf{t}_{i k}
$$

with the explicit expression of the $\beta$ coefficients

$$
\beta_{i j k}=-\frac{\rho_{i k}}{\rho_{i j}} \frac{\left|\mathbf{B}_{i} \mathbf{B}_{j}\right|}{\left|\mathbf{B}_{i} \mathbf{B}_{k}\right|}
$$




\subsubsection{The slope limiter}

We now focus on the reconstruction of $U_{i j}$. To this end, we consider the physical variable vector

$$
V=\left(\begin{array}{c}
\rho \\
u_{r} \\
u_{z} \\
u_{\theta} \\
P
\end{array}\right),
$$

and denote by $v$ a generic component of the vector $V$, i.e. $v=\rho, u_{r}, u_{z}, u_{\theta}$ or $P$. We construct a first set of slopes, that will be referred to as downstream slopes, and defined by

$$
p_{i j}^{+}=\frac{v_{j}-v_{i}}{\left|\mathbf{B}_{i} \mathbf{B}_{j}\right|} \quad \text { for all } C_{i} \in \mathcal{T}_{h}, j \in \nu(i) .
$$

The slopes represent an approximation of the directional derivative following $\mathbf{t}_{i j}$. Obviously, we have to construct five slopes $p_{i j}^{+}$, one for each variable. We define the upstream slopes by

$$
p_{i j}^{-}=\sum_{\substack{k \in \nu(i) \\ k \neq j}} \beta_{i j k} p_{i k}^{+} \quad \text { for all } C_{i} \in \mathcal{T}_{h}, j \in \nu(i) .
$$

We finally compute the slopes $p_{i j}$ using a limiting procedure, for example

$$
p_{i j}=\operatorname{minmod}\left(p_{i j}^{+}, p_{i j}^{-}\right) \text {. }
$$

We eventually construct the new value $v_{i j}$ by setting

$$
v_{i j}=v_{i}+p_{i j}\left|\mathbf{B}_{i} \mathbf{Q}_{i j}\right|
$$

With $V_{i j}$ and $V_{j i}$ in hand, we compute the conservative variable vector $U_{i j}$ and $U_{j i}$ on both sides of the interface $S_{i j}$ to provide the flux evaluation $\mathcal{F}\left(U_{i j}, U_{j i}, \mathbf{n}_{i j}\right)$.

Remark 1 Thanks to the fundamental decomposition (19) we can prove that this reconstruction is exact for linear functions, i.e. $v\left(Q_{i j}\right)=v_{i j}$, if $v$ is piecewise linear. 
Remark 2 The positivity of the barycentric coordinates implies that $\beta_{i j k}<0$. An important consequence is that if $v_{i}$ is a local extremum then $p_{i j}^{+} p_{i j}^{-} \leq 0$. This implies $p_{i j}=0$, which prevents the extrema from increasing (see [13]).

Remark 3 The main issue is that we consider only one-dimensional reconstruction in each direction. This enables using any classical 1-D limiter to compute the slope $p_{i j}$ (see [14] for more details). In the case where $C_{i}$ has a common edge with the boundary, we set $p_{i j}=0$ which results in a first-order scheme.

Remark 4 It is important to perform the reconstruction with the physical variables instead of the conservative variables. Indeed, in some situations a reconstruction based on the conservative variables can yield a negative internal energy since it is obtained by subtracting the kinetic energy from the total energy.

\section{Steady-state radial solutions}

Let us investigate the particular case of steady-state radial solutions. This case is helpful in the sense that it provides a reference solution to test the chosen numerical scheme. In practice, a stationary solution is obtained by supplying adequate boundary conditions to the time dependent problem and letting the solution converge to a stationary one. In addition, as far as some compressible flows for industrial applications like in induction plasma problems (see [19] for instance) are concerned, stationary solutions are to be considered.

It turns out that it is rather difficult to compute an exact solution for stationary flows. To provide a simple case, we resort then to restrict this section to radial solutions, i.e. that do not depend on $z$ and such that $u_{z}=0$. Under these assumptions, equations (10)-(14) reduce to:

$$
\begin{aligned}
& \frac{d}{d r}\left(r \rho u_{r}\right)=0, \\
& \frac{d}{d r}\left(r\left(\rho u_{r}^{2}+P\right)\right)=\rho u_{\theta}^{2}+P, \\
& \frac{d}{d r}\left(\rho u_{\theta} u_{r}\right)=-\rho u_{\theta} u_{r}, \\
& \frac{d}{d r}\left(r u_{r}(E+P)\right)=0 .
\end{aligned}
$$

Using the state equation of an ideal gas (4), the integration of equations (21), (23) and (24) gives: 


$$
\begin{aligned}
& \rho u_{r}=\frac{\alpha_{1}}{r}, \\
& \rho u_{\theta} u_{r}=\frac{\alpha_{2}}{r^{2}}, \\
& u_{r}\left(\frac{\gamma}{\gamma-1} P+\frac{1}{2} \rho\left(u_{r}^{2}+u_{\theta}^{2}\right)\right)=\frac{\alpha_{3}}{r},
\end{aligned}
$$

where $\alpha_{1}, \alpha_{2}$ and $\alpha_{3}$ are constants.

Thanks to relations (25) and (26), the expression of the tangential velocity $u_{\theta}$ becomes

$$
u_{\theta}=\frac{\alpha_{2}}{\alpha_{1} r} .
$$

Let us introduce two new variables: $a=\rho u_{r}^{2}$ and $b=\rho u_{\theta}^{2}$. The system becomes then:

$$
\left\{\begin{array}{l}
a b=\frac{\alpha_{2}^{2}}{r^{4}} \\
\frac{\gamma}{\gamma-1} P+\frac{1}{2}(a+b)=\frac{\alpha_{1} \alpha_{3}}{a r^{2}} \\
\frac{d(a+P)}{d r}=\frac{b-a}{r}
\end{array}\right.
$$

We now show how to reduce system (29) to an ordinary differential equation that we solve numerically. We first deal with the simple case where $u_{\theta}=0$, i.e. $b=0$. Then, we consider the more general case of a swirling flow.

\subsection{First case: $u_{\theta}=0$}

Let us first consider the case where the fluid flow has the property $u_{\theta}=0$. Then system (29) is reduced to:

$$
\left\{\begin{array}{l}
\frac{\gamma}{\gamma-1} P+\frac{1}{2} a=\frac{\alpha_{1} \alpha_{3}}{a r^{2}} \\
\frac{d(a+P)}{d r}=-\frac{a}{r}
\end{array}\right.
$$

From this we deduce

$$
\frac{d}{d r}\left(\frac{\gamma-1}{\gamma} \frac{\alpha_{1} \alpha_{3}}{a r^{2}}+\frac{\gamma+1}{2 \gamma} a\right)=-\frac{a}{r}
$$


Therefore

$$
\frac{d a}{d r}=-\frac{a}{r} \frac{2 A-1}{A-\frac{\gamma+1}{2 \gamma}}
$$

where

$$
A=\frac{\gamma-1}{\gamma} \frac{\alpha_{1} \alpha_{3}}{a^{2} r^{2}}=\frac{\gamma-1}{\gamma} \frac{\alpha_{3}}{\alpha_{1}^{3}} r^{2} \rho^{2}
$$

By differentiating the identity $\alpha_{1}^{2}=r^{2} \rho a$, we deduce

$$
\frac{d \rho}{d r}=-\alpha_{1}^{2}\left(\frac{2 a+r a^{\prime}}{r^{3} a^{2}}\right)
$$

From (31), we get

$$
\frac{d \rho}{d r}=\frac{\alpha_{1}^{2}}{\gamma a r^{3}}\left(\frac{1}{A-\frac{\gamma+1}{2 \gamma}}\right)
$$

This eventually gives the ordinary differential equation for the density:

$$
\frac{d \rho}{d r}=\frac{\rho}{\left(\frac{\alpha_{3}}{\alpha_{1}^{3}} \rho^{2} r^{2}-\frac{\gamma+1}{2(\gamma-1)}\right)(\gamma-1) r}
$$

The numerical solution of equation (32) is described in section 4.3.

Remark 5 An analytical solution of equation (32) can be obtained if we assume that

$$
\frac{\alpha_{3}}{\alpha_{1}^{3}} \rho^{2} r^{2} \gg \frac{\gamma+1}{2(\gamma-1)}
$$

This assumption is valid in particular for subsonic flows at ambient temperature for instance. Neglecting the higher order term, the differential equation (32) reduces to

$$
2 \rho \frac{d \rho}{d r}=\frac{\alpha_{1}^{3}}{(\gamma-1) \alpha_{3}} \frac{2}{r^{3}}
$$


Using the initial condition $\rho\left(r=R_{0}\right)=\rho_{0}$, we obtain the solution

$$
\rho(r)=\sqrt{\rho_{0}^{2}+\frac{\alpha_{1}^{3}}{\alpha_{3}(\gamma-1)}\left(\frac{1}{R_{0}^{2}}-\frac{1}{r^{2}}\right)} .
$$

\subsection{The general case (swirling flow)}

We now deal with the general case where $u_{\theta} \neq 0$. Differentiating the first equation of system (29) yields

$$
b \frac{d a}{d r}+a \frac{d b}{d r}=-\frac{4 a b}{r} .
$$

Thus

$$
\frac{d b}{d r}=-\frac{b}{a} \frac{d a}{d r}-\frac{4 b}{r} .
$$

Let us substitute $P$ in the system (29) and use (33) and (34). We get

$$
\frac{d}{d r}\left(\frac{\gamma-1}{\gamma} \frac{\alpha_{1} \alpha_{3}}{a r^{2}}-\frac{\gamma-1}{2 \gamma} b+\frac{\gamma+1}{2 \gamma} a\right)=\frac{b-a}{r} .
$$

We compute the derivative of the previous expression and eliminate the derivative of $b$ by using (34),

$$
\frac{\gamma-1}{\gamma a}\left(\frac{b}{2}-\frac{\alpha_{1} \alpha_{3}}{a r^{2}}\right) \frac{d a}{d r}=\frac{a}{r}\left(\frac{b}{a}-\frac{\gamma-1}{\gamma a}\left(\frac{4 b}{2}-\frac{2 \alpha_{1} \alpha_{3}}{a r^{2}}-1\right)\right) .
$$

Since $\alpha_{1}^{2}=r^{2} \rho a$, we obtain

$$
\frac{d \rho}{d r}=-C_{1}^{2}\left(\frac{2 r a+r^{2} \frac{d a}{d r}}{r^{4} a^{2}}\right)
$$

After calculation, the two previous equations give:

$$
\frac{d \rho}{d r}=-\frac{\alpha_{1}^{2}}{a r^{3}}\left(\frac{a+b}{(\gamma-1)\left(\frac{b}{2}-\frac{\alpha_{1} \alpha_{3}}{a r^{2}}\right)+\frac{(\gamma+1)}{2} a}\right)
$$


Using the definitions of $\rho$ and $P$, we finally obtain the ordinary differential equation for the fluid density:

$$
\frac{d \rho}{d r}=\frac{\rho}{r}\left(\frac{a+b}{\gamma P-a}\right) .
$$

Remark 6 In equation (35), functions $a, b, P$ depend only on $\rho, r$ and the constants. Indeed, for a given $\rho$, we can compute $b=\rho u_{\theta}^{2}$ using (28). We then deduce a with the first relation of system (29). We finally compute $P$ with the help of the second relation of system (29).

\subsection{A numerical method for stationary radial solutions}

We consider a uniform subdivision of the domain $\left[R_{0}, R_{1}\right]$ with $K$ elements where $r_{k}=R_{0}+k \Delta r, k=0, \ldots, K$ with $\Delta r=\left(R_{1}-R_{0}\right) / K$. To avoid the singularity at $r=0$, we have taken $R_{0}>0$. To obtain an approximation of the density $\rho$ for the equations (32) and (35), we use the explicit Euler method:

$$
\begin{array}{ll}
\text { case } u_{\theta}=0: & \rho_{k+1}=\rho_{k}+\Delta r \frac{\rho_{k}}{\left(\frac{\alpha_{3}}{\alpha_{1}^{3}} \rho_{k}^{2} r_{k}^{2}-\frac{\gamma+1}{2(\gamma-1)}\right)(\gamma-1) r_{k}}, \\
\text { case } u_{\theta} \neq 0: & \rho_{k+1}=\rho_{k}+\Delta r \frac{\rho_{k}}{r_{k}}\left(\frac{a_{k}+b_{k}}{\gamma P_{k}-a_{k}}\right) .
\end{array}
$$

The other parameters, namely the radial velocity $u_{r}$, the tangential velocity $u_{\theta}$ and the pressure $P$, are deduced using:

$$
\begin{aligned}
& u_{r, k+1}=\frac{\alpha_{1}}{\rho_{k+1} r_{k+1}}, \\
& u_{\theta, k+1}=\frac{\alpha_{2}}{\alpha_{1} r_{k+1}}, \\
& P_{k+1}=\frac{\gamma-1}{\gamma}\left(\frac{\alpha_{1} \alpha_{3}}{\rho_{k+1} u_{r, k+1}^{2} r_{k+1}^{2}}-\frac{1}{2} \rho_{k+1}\left(u_{r, k+1}^{2}+u_{\theta, k+1}^{2}\right)\right) .
\end{aligned}
$$

The $a$ and $b$ values are then updated by relations:

$$
\begin{aligned}
& a_{k+1}=\rho_{k+1} u_{r, k+1}^{2}, \\
& b_{k+1}=\rho_{k+1} u_{\theta, k+1}^{2},
\end{aligned}
$$




\section{$5 \quad$ Numerical tests}

We present several numerical tests to show convergence and accuracy properties of the MUSCL scheme presented throughout this paper. The first test shows that the algorithm preserves the situation when the gas is at rest which proves that the scheme is well-balanced in this sense.

A second series of tests concerns radial steady-state solutions. We observe convergence for large time to the stationary solution computed with the numerical method proposed in subsection 4.3 .

A third series of numerical experiments is dedicated to the Riemann problem. We consider a domain $\Omega=[0,1] \times[0,1]$ and impose an initial condition made of two constant states for $z<1 / 2$ and $z>1 / 2$. We obtain a classical onedimensional Sod tube problem in the $O z$ direction. We then compare the numerical solutions to the analytical ones.

Finally, we describe two more significant test problems. The first one is an adaption of the well-known front-facing step problem of [20] for an axisymmetric configuration where a stationary Mach 3 flow hits a step. The second test produces a converging spherical shock simulating a gas bubble compression. This test problem allows to check the capacity of the numerical scheme to preserve the spherical symmetry of the problem.

Computations have been carried out using the finite element / finite volume library OFELI described in [21]. We detail all these numerical experiments in the sequel.

\subsection{Conservation of the gas at rest}

In this test, we check the capacity of the algorithm to preserve a situation where the gas is at rest. We consider a domain $\Omega=[0,1] \times[0,1]$ and prescribe the following initial conditions:

$$
u_{r}=u_{\theta}=u_{z}=0, \quad P=1 \times 10^{5} \mathrm{~Pa}, \quad \rho=1.2 \mathrm{~kg} \cdot \mathrm{m}^{-3}
$$

We also consider a second test where the initial null axial velocity is given by $u_{z}=100 \mathrm{~m} \cdot \mathrm{s}^{-1}$.

On the boundaries $r=0$ and $r=1$ we impose a reflection condition using the ghost-cell technique [16] while we enforce a Dirichlet condition for the boundaries $z=0$ and $z=1$ equal to the initial condition. 

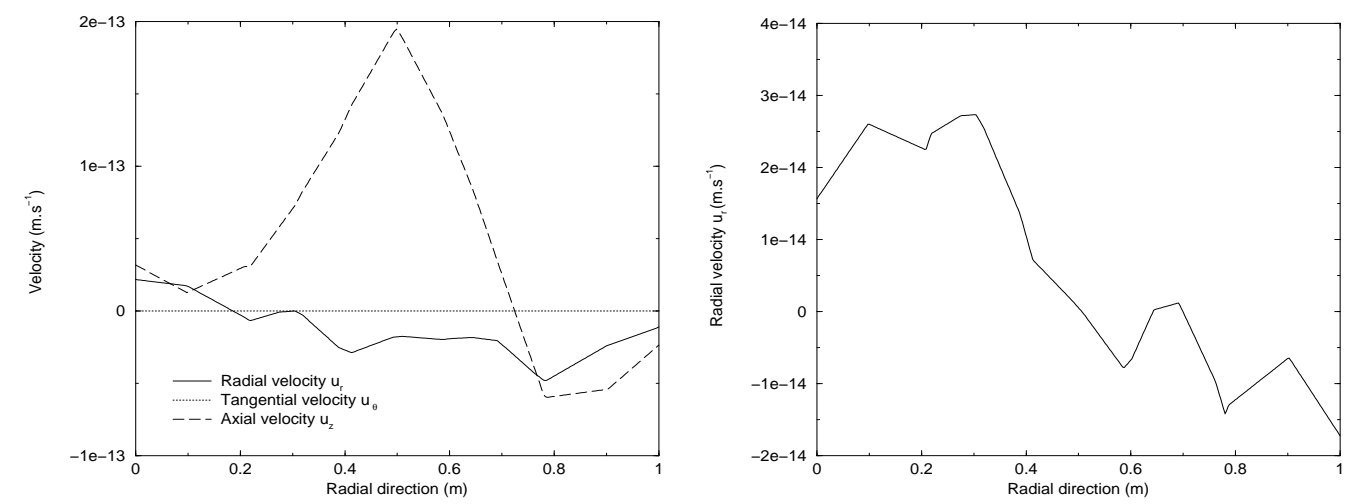

Fig. 2. Radial, axial and tangential velocity distribution following the radial direction at $z=0.5$ for the first test (Left) and radial velocity following the radial direction at $z=0.5$ for the second test (Right).

In both situations, we observe the preservation of the gas at rest. A few numerical artifacts of order $10^{-14}$ which correspond to the precision of the computer are present.

\subsection{Stationary solution}

The goal of the study is to consider steady-state radial solution approximations and show that any non-stationary solution of the system (15) using judicious boundary conditions $U_{\text {inlet }}$ and $U_{\text {outlet }}$ converges asymptotically to the stationary solution given in section 4 .

To compute the stationary solution, we use a subdivision following the onedimensional radial direction $\left(R_{0}=0.1, R_{1}=0.4\right)$ of 10000 cells. Since the problem reduces to an ordinary differential equation, we prescribe the initial condition at point $R_{0}$ (named the inlet condition $U_{\text {inlet }}$ ). Using the algorithm given in subsection 4.3, we obtain a numerical approximation on domain $\left[R_{0}, R_{1}\right]$ and we denote by $U_{\text {outlet }}$ the solution at point $R_{1}$.

To compute the non-stationary solution, we use the rectangular domain $\Omega=$ $[0.1,0.4] \times[0,0.1]$ discretized in 6800 triangle cells (approximately 100 cells along the radial direction). We prescribe the reflection condition at the boundaries $z=0$ and $z=0.1$ while we impose the inlet and outlet conditions at the boundaries $r=R_{0}$ and $r=R_{1}$ respectively. We initialize the solution with the inlet condition. Two numerical experiments have been performed, the first one concerns the simple case where $u_{\theta}=0$. The second test deals with the general case which corresponds to the swirling flow. 


\subsubsection{Case $u_{\theta}=0$}

We use the numerical scheme (36) and impose the following inlet conditions at $r=R_{0}$ :

$$
C_{\text {inlet }}=\left(\begin{array}{c}
\rho_{\text {inlet }}=2 \\
u_{r, \text { inlet }}=200 \\
u_{\theta, \text { inlet }}=0 \\
u_{z, \text { inlet }}=0 \\
P_{\text {inlet }}=2 \times 10^{5}
\end{array}\right)
$$

We compute all the variables of vector $U$ at each point of the subdivision, in particular we obtain the outlet condition at $r=R_{1}$

$$
C_{\text {outlet }}=\left(\begin{array}{c}
\rho_{\text {outlet }}=2.283222 \\
u_{r, \text { outlet }}=43.79776 \\
u_{\theta, \text { outlet }}=0 \\
u_{z, \text { outlet }}=0 \\
P_{\text {outlet }}=2.407435 \times 10^{5}
\end{array}\right) \text {. }
$$

We now introduce the two boundary conditions in the non-stationary scheme and run until a stationary situation is achieved.

In figure 3, we show the density, the pressure and the radial velocity in function of $r$ at $z=0.05$. We also propose a zoom of the density distribution to highlight the difference between the Rusanov and the HLLC solver but also between the first and the second order scheme. These two solutions clearly coincide and as we can expect, the second order scheme with the HLLC flux provides the best approximation.

\subsubsection{Case $u_{\theta} \neq 0$}

We now deal with the swirling flow where we use the algorithm (37) and the new inlet conditions: 

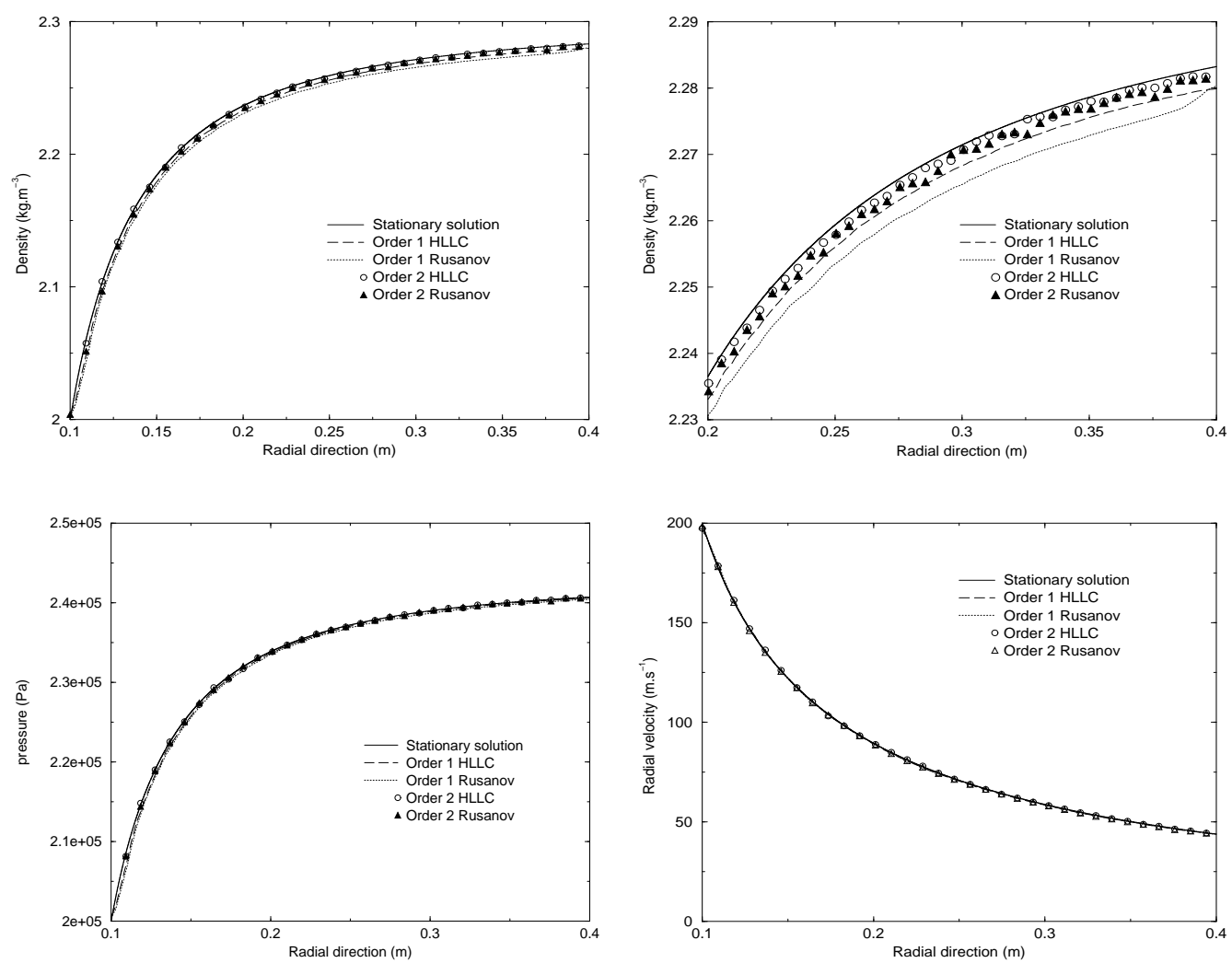

Fig. 3. Comparison of density, pressure and the radial velocity distributions versus the radial direction between the stationary solution and numerical results.

$$
C_{\text {inlet }}=\left(\begin{array}{c}
\rho_{\text {inlet }}=2 \\
t u_{r, \text { inlet }}=200 \\
u_{\theta, \text { inlet }}=10 \\
u_{z, \text { inlet }}=0 \\
P_{\text {inlet }}=2 \times 10^{5}
\end{array}\right)
$$

We also provide the outlet condition:

$$
C_{\text {outlet }}=\left(\begin{array}{c}
\rho_{\text {outlet }}=2.283956 \\
u_{r, \text { outlet }}=43.78367 \\
u_{\theta, \text { outlet }}=2.5 \\
u_{z, \text { outlet }}=0 \\
P_{\text {outlet }}=2.408519 \times 10^{5}
\end{array}\right) \text {. }
$$


With the two boundary conditions in hand, we compute the non-stationary solution until a stationary solution is obtained.
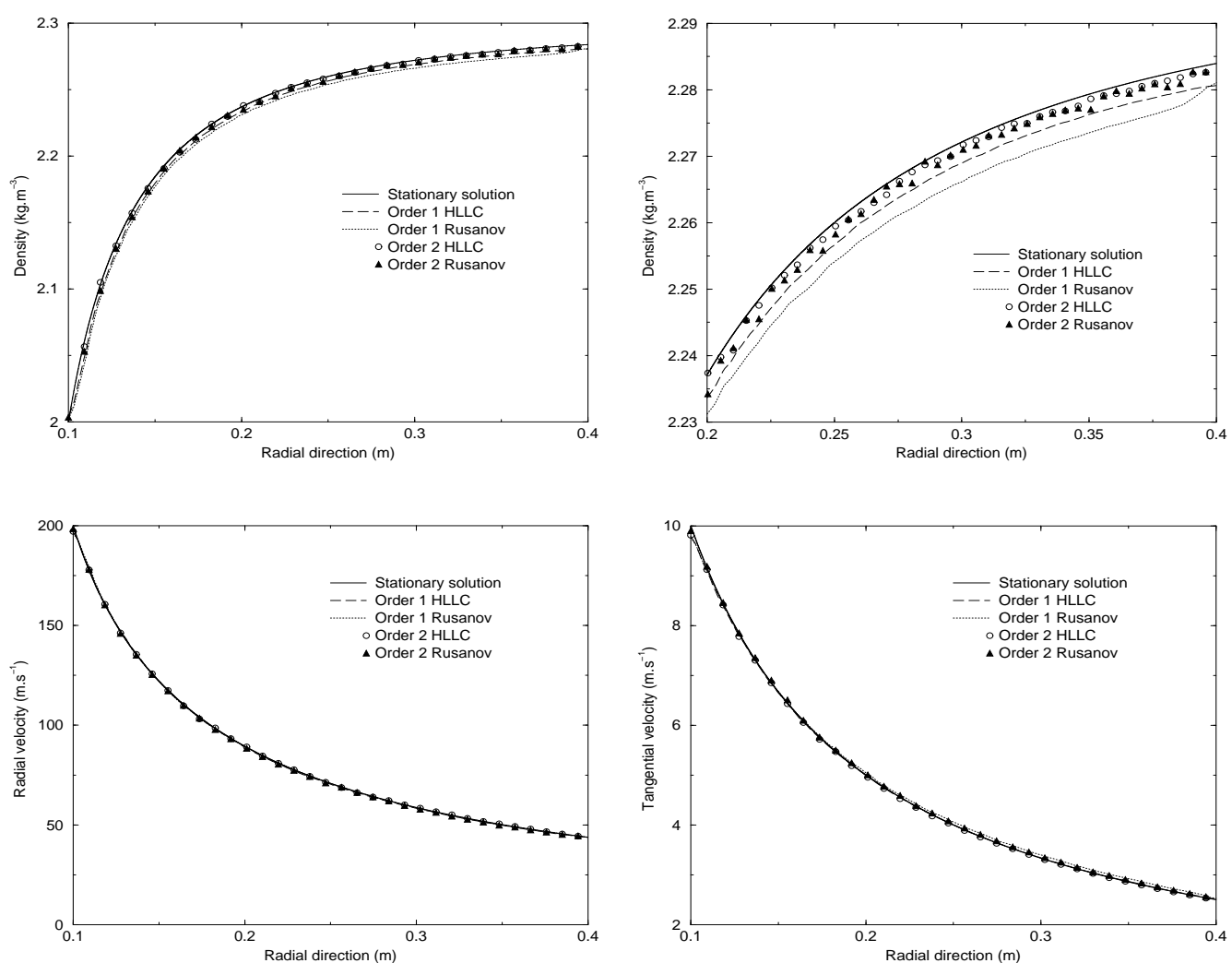

Fig. 4. Comparison of density, radial and tangential velocity distributions versus the radial direction between the stationary solution and numerical results.

Figure 4 shows density and velocity components $u_{r}$ and $u_{\theta}$. We also include a zoom of the density to highlight the second order scheme efficiency. As in the previous case, we succeed in reaching the steady-state solution and the second order scheme with the HLLC flux provides the best solution.

\subsection{A shock tube test}

We decompose the domain $\Omega=[0,1] \times[0,1]$ into two subdomains $\Omega_{L}=$ $[0,1] \times\left[0, \frac{1}{2}\right]$ and $\Omega_{R}=[0,1] \times\left[\frac{1}{2}, 1\right]$ and denote by $D$ the line $D=\left\{\left(r, \frac{1}{2}\right) ; r \in\right.$ $[0,1]\}$. We then consider the Sod tube problem prescribing the initial condition $U(t=0)=U_{L}$ in $\Omega_{L}$ and $U(t=0)=U_{R}$ in $\Omega_{R}$. We also impose the reflection condition at the boundary of $\Omega$. It results that the solution is invariant with respect to $r$ and we obtain a classical one-dimensional Sod tube problem in the $O z$ direction with an initial discontinuity at $z=1 / 2$. 
We compare exact solutions of the Riemann problem to the numerical approximations obtained using the first and second-order schemes and the Rusanov and HLLC schemes. All the computations have been performed with an unstructured mesh of 20748 elements.

\subsubsection{First configuration: Rarefaction and shock}

We consider a first configuration providing a rarefaction for the $u-c$ characteristic field and a shock for the $u+c$ characteristic field. We denote by $U_{L}^{*}$, the state just after the rarefaction while $U_{R}^{*}$ represents the state just before the shock. Of course, a contact discontinuity occurs between the two intermediate states. We sum up in table 1 the used values in the test.

We solve the Riemann problem with the algorithm for axisymmetric configu-

Table 1

Data for the first Riemann problem

\begin{tabular}{|c|c|c|c|}
\hline & $\rho\left(\mathrm{kg} \cdot \mathrm{m}^{-3}\right)$ & $u_{z}\left(\mathrm{~m} \cdot \mathrm{s}^{-1}\right)$ & $\mathrm{P}(\mathrm{Pa})$ \\
\hline \hline$U_{L}$ & 4.0 & 0.0 & $4.0 \times 10^{5}$ \\
\hline$U_{L}^{*}$ & 2.272739 & 200.0 & $1.81278 \times 10^{5}$ \\
\hline$U_{R}^{*}$ & 1.363643 & 200.0 & $1.81278 \times 10^{5}$ \\
\hline$U_{R}$ & 0.950418 & 47.949324 & $1.08767 \times 10^{4}$ \\
\hline
\end{tabular}

rations using the Rusanov and HLLC flux and using the first and second order techniques. We present in figure 5 a comparison of the density and internal
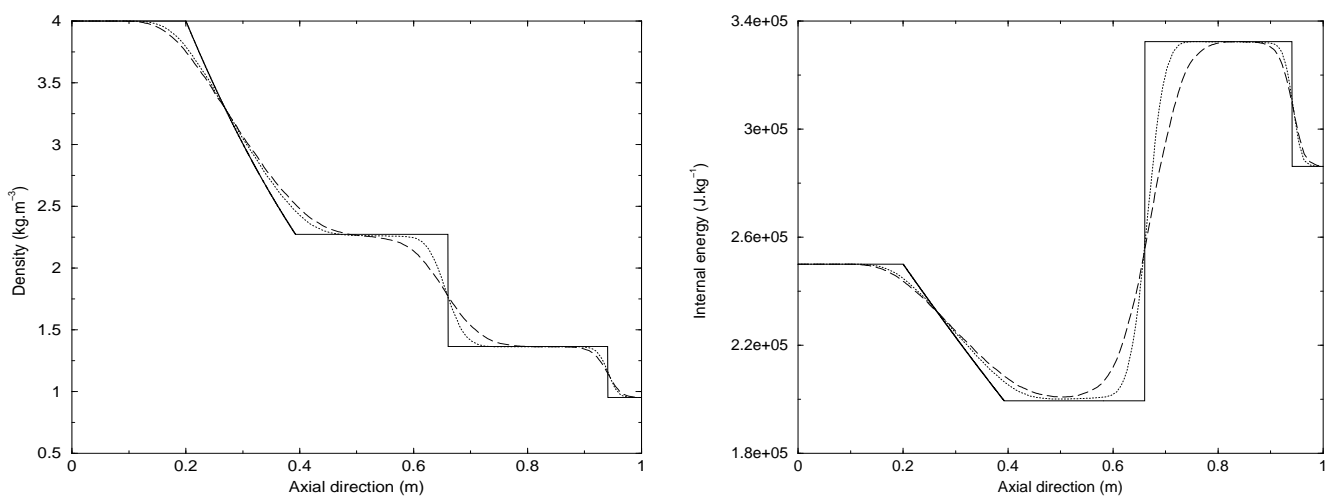

Fig. 5. Comparison of density and internal energy obtained by first-order Rusanov scheme (dashed) and first-order HLLC scheme (dotted) with the exact solution (continuous) at time $t=0.8 \mathrm{~ms}$ for the first configuration.

energy between the exact solution and the approximations using the Rusanov and HLLC flux with the first order scheme. In figure 6, a similar comparison is shown but we use the second order MUSCL technique. The solutions are 

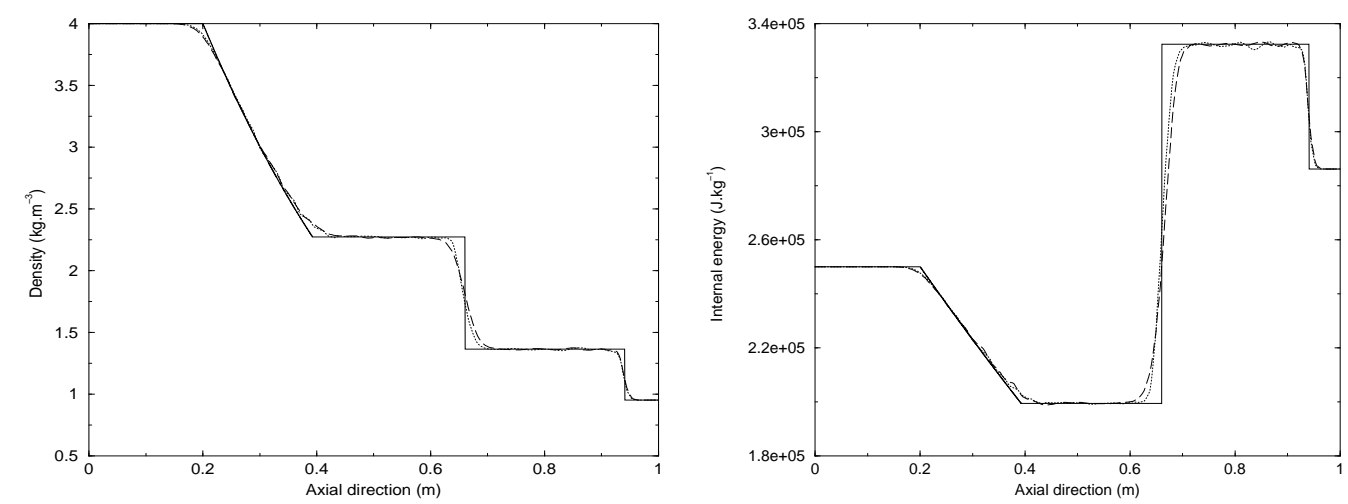

Fig. 6. Comparison of density and internal energy obtained by second-order Rusanov scheme (dashed) and second-order HLLC scheme (dotted) with the exact solution (continuous) at time $t=0.8 \mathrm{~ms}$ for the first configuration.

in good agreement with the exact solution and the second order technique provides the best approximations.

\subsubsection{Second configuration: double shock}

Here; we are concerned with the double shock configuration. Table 2 lists the states we employ in this test.

Table 2

Data for the second Riemann problem

\begin{tabular}{|c|c|c|c|}
\hline & $\rho\left(\mathrm{kg} \cdot \mathrm{m}^{-3}\right)$ & $u_{z}\left(\mathrm{~m} \cdot \mathrm{s}^{-1}\right)$ & $P(\mathrm{~Pa})$ \\
\hline \hline$U_{L}$ & 4.2 & 200.0 & $1.0 \times 10^{5}$ \\
\hline$U_{L}^{*}$ & 8.866667 & 41.688103 & $3.0 \times 10^{5}$ \\
\hline$U_{R}^{*}$ & 7.093333 & 41.688103 & $3.0 \times 10^{5}$ \\
\hline$U_{R}$ & 1.860546 & -285.504113 & $3.0 \times 10^{4}$ \\
\hline
\end{tabular}

We compute the solution approximations with the algorithm dedicated to axisymmetric geometries and visualize the density and the internal energy using the first order scheme (figure 7 ) or the second order scheme (figure 8). As expected, the MUSCL technique reduces the diffusion effect close to the discontinuities. We also remark that the Rusanov and HLLC schemes provide the same approximation quality in the second order context whereas the HLLC flux is less diffusive if one uses a first-order scheme. 

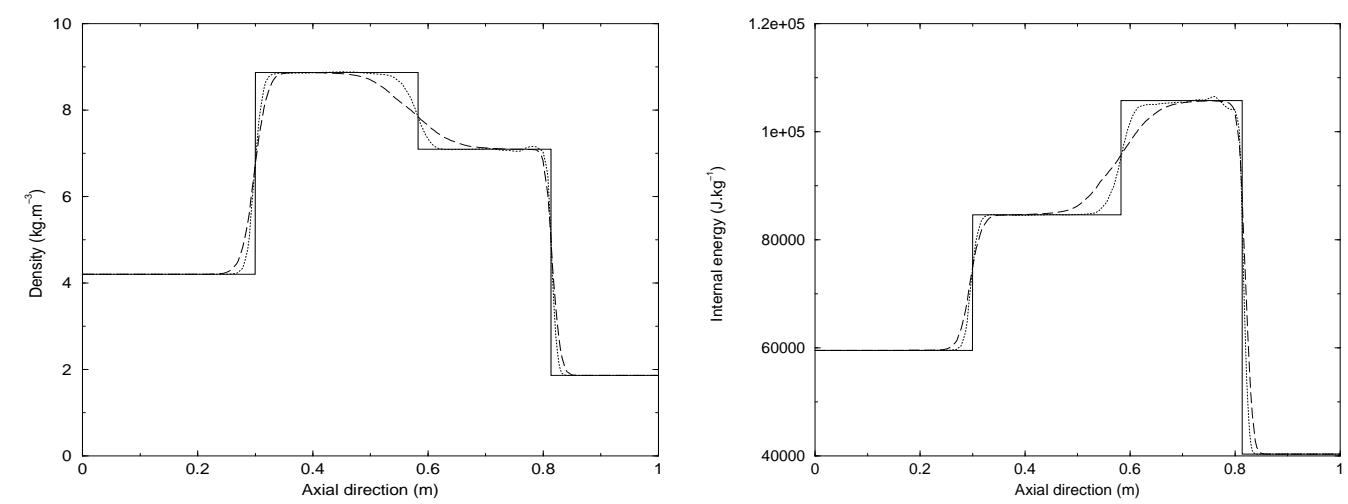

Fig. 7. Comparison of density and internal energy obtained by first-order Rusanov scheme (dashed) and a first-order HLLC scheme (dotted) with the exact solution (continuous) at time $t=1.984 \mathrm{~ms}$ for the second configuration.
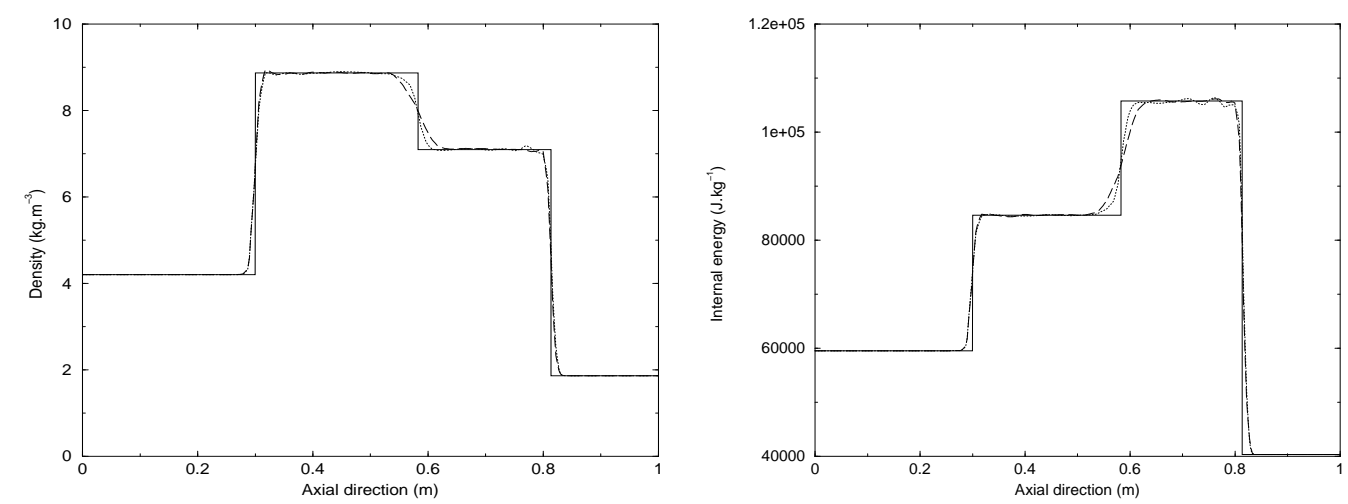

Fig. 8. Comparison of density and internal energy obtained by a second-order Rusanov scheme (dashed) and a second-order HLLC scheme (dotted) with the exact solution (continuous) at time $t=1.984 \mathrm{~ms}$ for the second configuration.

\subsubsection{Third configuration: double rarefaction}

The third configuration is composed of two symmetric rarefaction waves where the solution involves a state near vacuum. We list in table 3 the states we use for the calculation.

Table 3

Data for the third Riemann problem

\begin{tabular}{|c|c|c|c|}
\hline & $\rho\left(\mathrm{kg} \cdot \mathrm{m}^{-3}\right)$ & $u_{z}\left(\mathrm{~m} \cdot \mathrm{s}^{-1}\right)$ & $P(\mathrm{~Pa})$ \\
\hline \hline$U_{L}$ & 1.0 & -300.0 & $1.0 \times 10^{5}$ \\
\hline$U_{L}^{*}$ & 0.417325 & 0.0 & $2.94 \times 10^{5}$ \\
\hline$U_{R}^{*}$ & 0.417325 & 0.0 & $2.94 \times 10^{5}$ \\
\hline$U_{R}$ & 1.0 & 300.0 & $1.0 \times 10^{5}$ \\
\hline
\end{tabular}


Approximations have been computed using the first (figure 9) and the second order technique (figure 10). The main issue is the internal energy approximation. First order methods give a non physical peak at $z=0.5$ while second order methods reduce this numerical artifact. We note that the HLLC flux with the MUSCL technique reduces significantly the undesired peak.
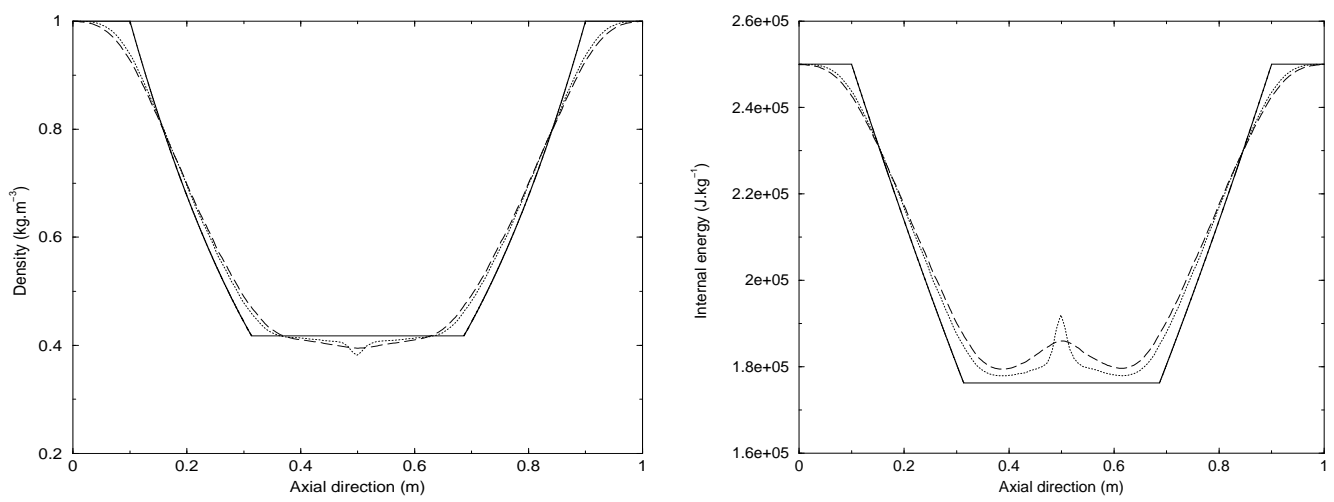

Fig. 9. Comparison of density and internal energy obtained by first-order Rusanov scheme (dashed) and first-order HLLC scheme (dotted) with the exact solution (continuous) at time $t=0.6 \mathrm{~ms}$ for the third configuration.
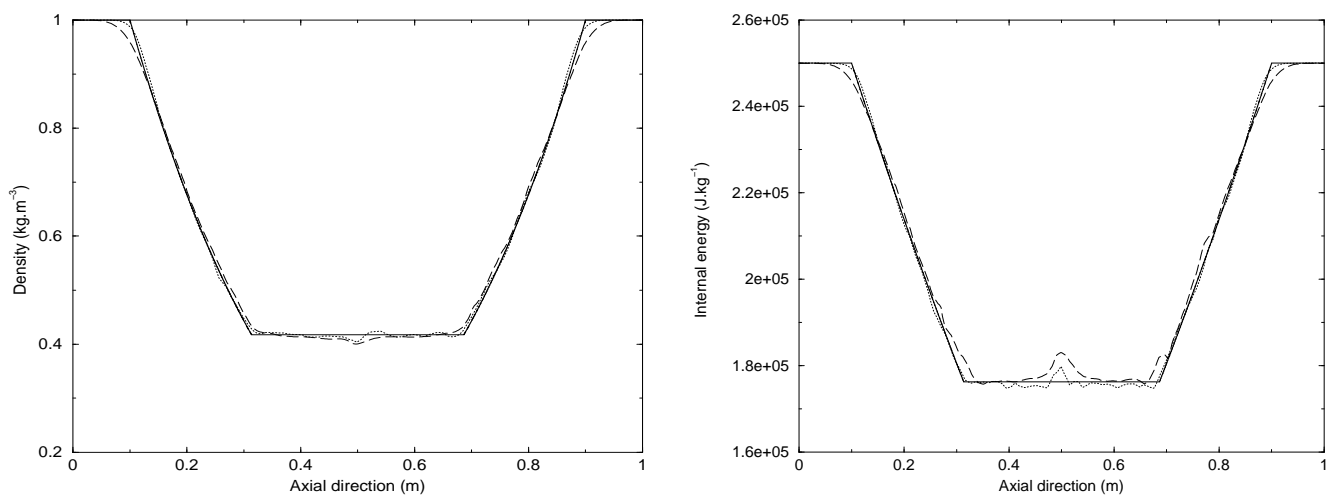

Fig. 10. Comparison of density and internal energy obtained by second-order Rusanov scheme (dashed) and second-order HLLC scheme (dotted) with the exact solution (continuous) at time $t=0.6 \mathrm{~ms}$ for the third configuration.

\subsection{A Mach 3 wind tunnel with a forward-facing step}

This numerical test deals with a Mach 3 flow in a wind tunnel with a forwardfacing step. This test is analogous to the well-known test problem of [20]. We have considered here a discretization of the domain into 21186 finite volume cells. A stable shock wave pattern develops after a few time units. We impose the inflow boundary conditions with $\rho=1.4, u_{z}=3, u_{r}=u_{\theta}=0$ and $P=1$. Figure 11 shows the snapshot of flow field at $t=15 \mathrm{~s}$. 

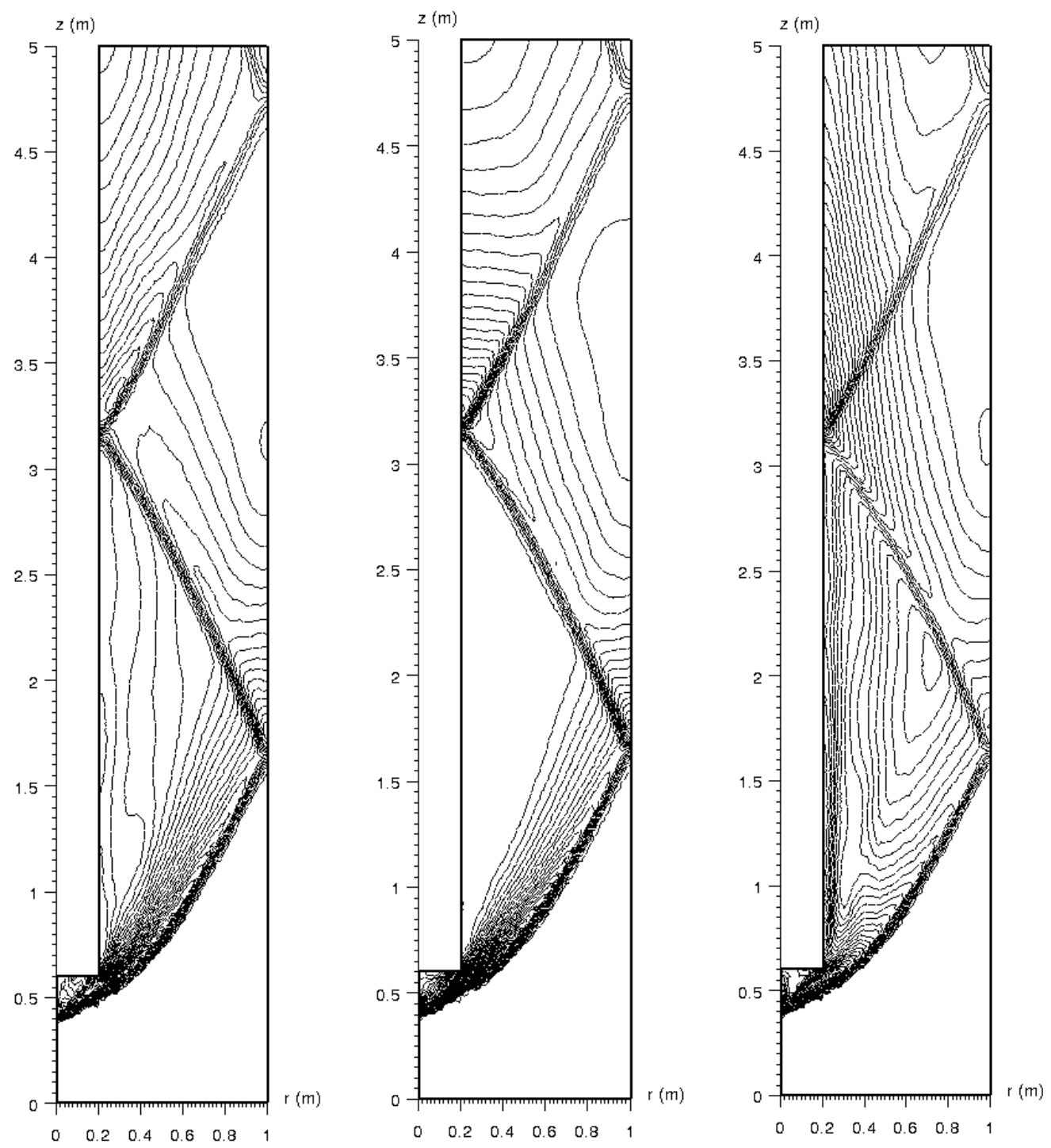

Fig. 11. Forward-facing step using a second-order HLLC scheme. 100 contours of the density (left) from 0.762 to 5 , pressure (centre) from 0.462 to 11.8 and Mach number (right) from 0.077 to 3.02 .

\subsection{Converging spherical shock test}

In $\mathbb{R}^{3}$, a spherical bubble of gas, with radius $R$, centered at the origin is compressed by an overpressured exterior gas of same nature. The solution involves the three classical waves and depends only on $R$, i.e. $U(r, z)=U(R, 0)=$ $U(0, R)$ for any point $(r, z)$ such that $R^{2}=r^{2}+z^{2}$.

To treat the spherical bubble problem with the axisymmetric model, we can choose arbitrarily the $O r$ and $O z$ axes, the main difficulty being that the variables $r$ and $z$ do not play the same role in the axisymmetric situation. The goal of this test is to study the asymmetry of the solution following the $\mathrm{Or}$ 
and $O z$ axis.

To simulate the spherical bubble compression using the axisymmetric model, we consider a quarter of disk $D=\left\{(r, z) ; \sqrt{r^{2}+z^{2}} \leq 1 / 2\right\}$ in the unit square $\Omega=[0,1] \times[0,1]$ and we state the following initial conditions:

$$
\left(\rho, u_{r}, u_{z}, u_{\theta}, P\right)=\left\{\begin{array}{l}
(1,0,0,0,1) \text { in } D, \\
(4,0,0,0,4) \text { in } \Omega \backslash D .
\end{array}\right.
$$

We also prescribe reflecting boundary conditions on the whole boundary. We have performed numerical tests with a discretization of the domain into 20640 finite volume cells.

In figure 12 we reproduce the density distribution at time $t=0.2$ using the Rusanov and the HLLC fluxes. A small asymmetry appears when the Rusanov flux is employed but it disappears when we use the MUSCL procedure. This is confirmed by the visualization of the density value along the $O r$ and $O z$ axis in figure 13 .

\section{Conclusion}

We have proposed a new second order cell-centered finite volume formulation for compressible Euler equations using cylindrical coordinates to compute axisymmetric solutions. The finite volume scheme is based on a mean value approximation using the $r d r d z$ metric instead of the traditional $d r d z$ metric to simplify the boundary condition on the $\mathrm{Or}$ axis. An original MUSCL method (multislope MUSCL method) is introduced where an estimate of the directional derivatives is performed to construct more accurate approximations of the solution on both side of the edges. New numerical tests based on the steady-state situation has been proposed to validate the numerical scheme and to compare the different schemes performances.

\section{References}

[1] P.A. Jacob, Transient, hypervelocity flow in an axisymmetric nozzle, ICATE report No 91-1, NASA research center (1991).

[2] M.A. Leschziner, W. Rodi, Computation of strongly swirling axisymmetric free jets, AIAA Journal, 22 (1984) 1742-1747.

[3] C.H. Wu, A general theory of three-dimensional flow in subsonic and supersonic turbomachine in radial, axial and mixed flow types, NACA TN 2604 (1952). 

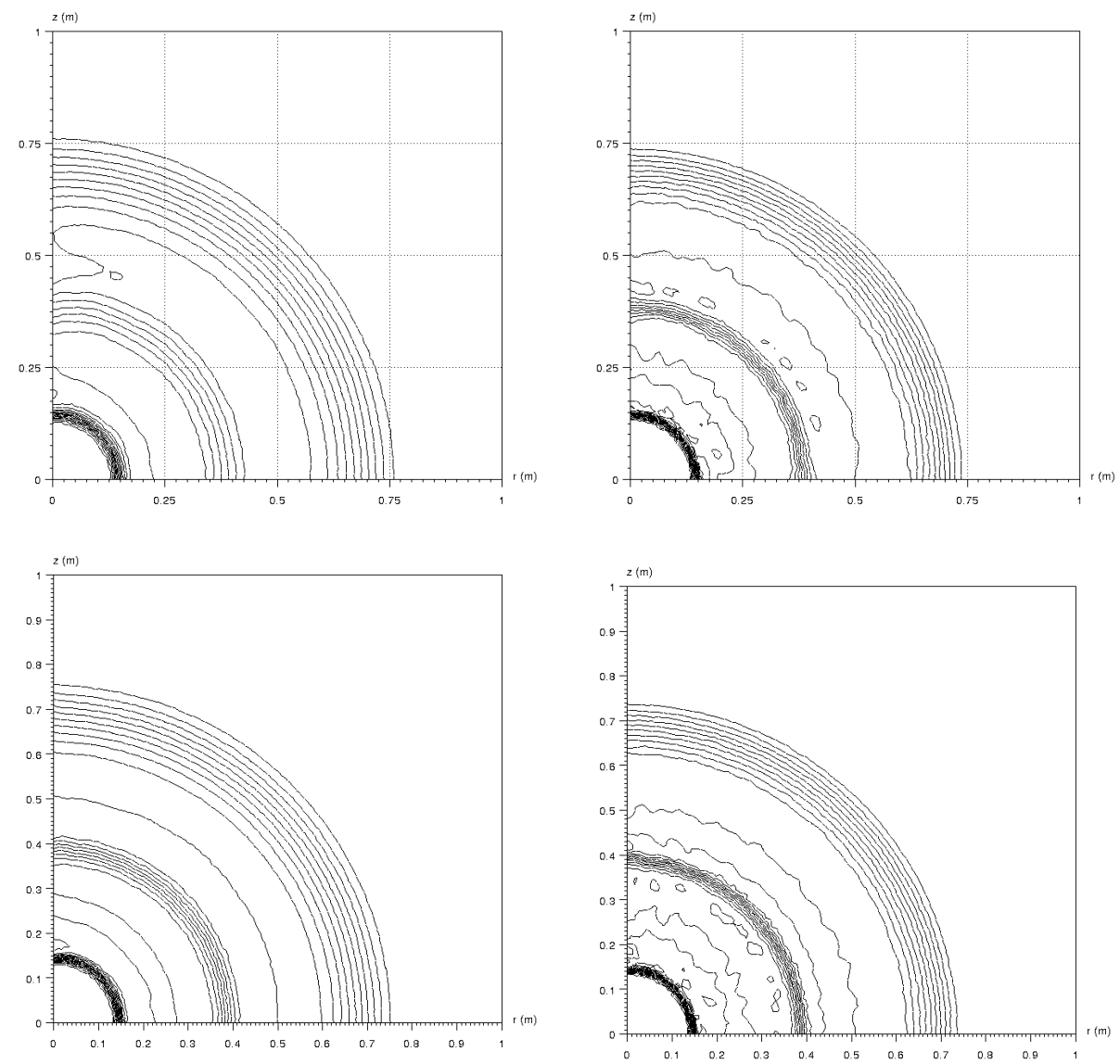

Fig. 12. Density at $t=0.2,31$ contours from 1 to 4 . Rusanov first-order (top left) and second-order (top right), HLLC first-order (bottom left) and second-order (bottom right).
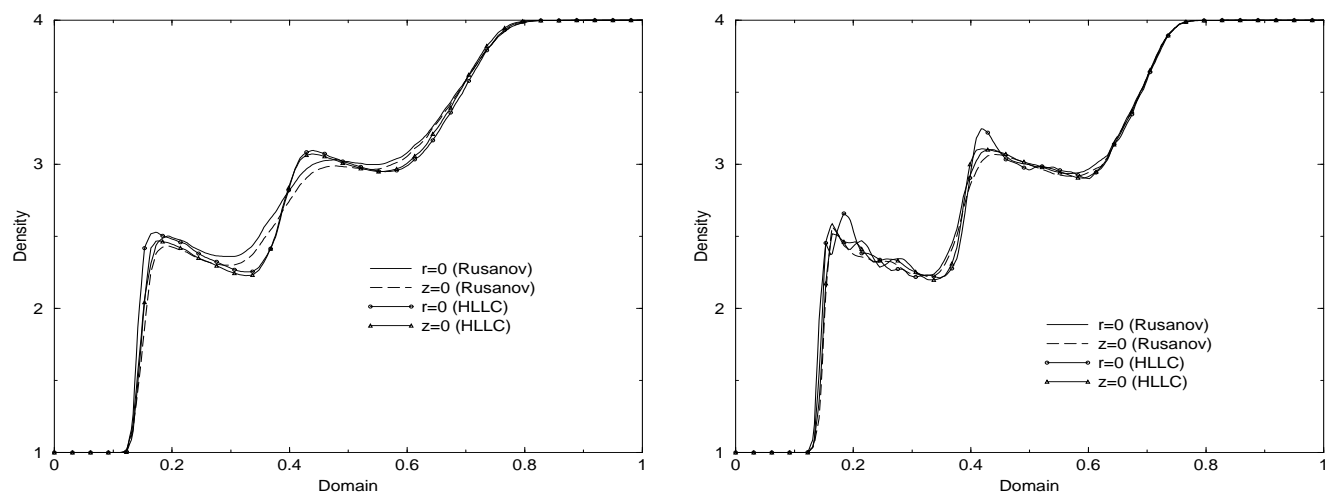

Fig. 13. Comparison of density at $t=0.2$ for $r=0$ and $z=0$ using first-order (Left) and second-order (Right) schemes. 
[4] Ch. Hirsch, G. Warzee, A finite element method for the axisymmetric flow computation in a turbomachine International Journal for Numerical Methods in Engineering, 10 (1) (1976) 93-113.

[5] D. Vanden Abeele, G. Degrez, An efficient computational model for inductive plasma flows, AIAA 2825 (1998).

[6] S. Xue, P. Proulx, M.I. Boulos, Extended-filed electromagnetic model for the inductively coupled plasma, J. Phys D: Appl. Phys. 34 (2001) 1897-1906.

[7] A. Guardone, L. Vigevano, Finite element/volume solution to axisymmetric conservation laws, Journal of Computational Physics, 224(2) (2007) 489-518.

[8] P. Glaister, Flux difference splitting for the Euler equations with axial symmetry, Journal of Engineering Mathematics 22 (1988) 107-121.

[9] J.A. Désidéri, A. Goudjo, Un schéma de volumes-finis décentré pour la résolution des équations d'Euler en axisymétrique, INRIA Report No. 1005 (1989).

[10] R. Cumming, H. Yang, Y. Oh, Supersonic, turbulent flow computation and drag optimization for axisymmetric afterbodies, Comput. Fluids, 24 (1995) 487-507.

[11] B. Van Leer, Towards the ultimate conservative difference scheme. V. A secondorder sequel to Godunov's method, Journal of Computational Physics, 32 (1) (1979) 101-136.

[12] M.E. Hubbard, Multidimensional Slope Limiters for MUSCL-Type Finite Volume Schemes on Unstructured Grids, J. Comp. Phys. 155 (1999) 54-74.

[13] S. Clain, V. Clauzon, The multislope MUSCL method, Proceeding in the Finite Volumes for Complex Application 5, Wiley, (2008) 297-304.

[14] V. Clauzon, Analyse de Schémas d'ordre élevé pour les écoulements compressibles. Application à la simulation numérique d'une torche à plasma, PhD Thesis, Blaise Pascal University, Clermont Ferrand, (2008), (Thesis available at http://tel.archives-ouvertes.fr/tel-00235951/fr/).

[15] T. Buffard, S. Clain, Monoslope and multislope MUSCL methods for unstructured meshes, submitted (2008), (Preprint available at http://hal .archives-ouvertes.fr/hal-00323691/fr/).

[16] E. F. Toro, Riemann Solvers and Numerical Methods for Fluid Dynamics, A practical Introduction, Springer-Verlag, Second Edition, Berlin (1999).

[17] P. Batten, N. Clarke, C. Lambert, D. M. Causon, on the choice of wavespeeds for the HLLC Riemann solver, SIAM J. Sci. Comput. 18 (6) (1997) 1553-1570.

[18] E. Godlewski, P.-A. Raviart, Hyperbolic systems of conservations laws, Applied Mathematical Sciences, Springer, Berlin (1995).

[19] S. A. Vasil'evskii, A. F. Kolesnikov, Numerical simulation of equilibrium induction plasma flows in a cylindrical plasmatron channel, Fluid Dynamics, 35 (2000) 769-777. 
[20] P. Woodward, P Colella, The Numerical Simulation of Two-Dimensional Fluid Flow with Strong Shocks, Journal of Computational Physics, 54 (1984) 115-173.

[21] R. Touzani, OFELI, An Object Finite Element Library, Copyright (C)1998-2008 Rachid Touzani, (http://www.ofeli.net/). 